Author's accepted preprint version 8.19.2021.

The version of record is:

Nees, M.A. (2021). Driver monitoring systems: Perceived fairness of consequences when distractions are detected. In Adjunct Proceedings of the 13th International Conference on Automotive User Interfaces and Interactive Vehicular Applications

(AutomotiveUI '21 Adjunct), September 09-14, 2021, Leeds, United Kingdom. https://doi.org/10.1145/3473682.3480264

\title{
Driver Monitoring Systems: Perceived Fairness of Consequences when Distractions are Detected
}

\author{
Michael A. Nees* \\ Lafayette College, neesm@lafayette.edu
}

Driver monitoring may become a standard safety feature to discourage distraction in vehicles with or without automated driving functions. Research to date has focused on technology for identifying driver distraction-little is known about how drivers will respond to monitoring systems. An exploratory online survey assessed the perceived risk and reasonableness associated with driving distractions as well as the perceived fairness of potential consequences when a driver monitoring system detects distractions under either manual driving or Level 2 automated driving. Although more research is needed, results suggested: (1) fairness was associated with perceived risk; (2) alerts generally were viewed as fair; (3) more severe consequences (feature lockouts, insurance reporting, automation lockouts, involuntary takeovers) generally were viewed as less fair; (4) fairness ratings were similar for manual versus Level 2 driving, with some potential exceptions; and (5) perceived risk of distractions was slightly lower with automated driving.

\section{CCS CONCEPTS - Human-centered computing $\rightarrow$ Interaction design $\rightarrow$ Empirical studies in interaction design}

Additional Keywords and Phrases: Driver monitoring, automated driving, driver distraction, risk perception

\section{ACM Reference Format:}

First Author's Name, Initials, and Last Name, Second Author's Name, Initials, and Last Name, and Third Author's Name, Initials, and Last Name. 2018. The Title of the Paper: ACM Conference Proceedings Manuscript Submission Template: This is the subtitle of the paper, this document both explains and embodies the submission format for authors using Word. In Woodstock '18: ACM Symposium on Neural Gaze Detection, June 03-05, 2018, Woodstock, NY. ACM, New York, NY, USA, 10 pages. NOTE: This block will be automatically generated when manuscripts are processed after acceptance.

\section{INTRODUCTION}

Skepticism has set in regarding the near-term viability of ubiquitous fully automated driving [11]. Instead, a protracted period of partially-automated driving seems likely, with human drivers expected to monitor automated functions and intervene during automation failures. Humans tend to perform poorly at vigilance monitoring tasks [21]. Further, research has shown that drivers expect to be able to engage in non-driving activities during periods of automation [15], and abuses of driving automation (e.g., engaging in prohibited non-driving behaviors) have been regularly documented [10].

One potential solution to managing driver distraction during periods of automation has been to pair partial automation with driver monitoring (DM). A DM system uses sensors and algorithms to detect when a driver is

\footnotetext{
* Place the footnote text for the author (if applicable) here.
} 
distracted (e.g., $[19,22]$ ) and can intervene to re-focus the driver (e.g., by providing an alert). An emerging consensus supports the importance of DM as a critical safety component in any automated driving system for which the driver is expected to actively oversee automated functions (see [16]). Even without automation, DM has been recognized as an important technology for improving safety on roads. In the United States, legislation has been introduced to require DM in all new vehicles [9], and similar initiatives are already in place in Europe [23]. Thus, DM appears poised to become a standard safety feature in all vehicles—not just vehicles with automated features.

To date, much research on DM functionality has focused on the development of the technology (sensors, machine learning algorithms, etc., see [8]) to ascertain driver states and behaviors. Less research has examined potential consequences after driver distraction is detected. Yet DM is a layer of automation that occurs in addition to the automation of driving functions per se-sensors and software automate the task of selfmonitoring one's own attention to the road, mental states, and engagement with in-vehicle distractions. As such, the effective design of DM will involve a host of human factors complications known to accompany automation, including acceptance, trust, complacency, overreliance, etc. (see [13]). Horrey et al. [7] noted that effective feedback and driver acceptance will be critical to the success of DM systems. A base of knowledge on design of feedback has shown that both concurrent and retrospective feedback can help to mitigate distracted driving in some cases (e.g., $[4,5,20]$ ), but fewer studies have been concerned with understanding acceptance of DM systems.

Related research on electronic performance monitoring in the workplace has shown that the perceived fairness of monitoring plays an important role in acceptance and performance [1,12], and this finding seems likely to extend to DM systems (see [6,7]). The current exploratory study used an online survey to examine the perceived fairness of several potential consequences for drivers when a DM system detects potential driver distractions. Nine different distractions were examined, and participants also were randomly assigned to read one of two different background descriptions of DM. The first description suggested that DM will accompany all driving (including manual driving) and made no mention of automation, whereas the second condition described DM as only accompanying engagement of Level 2 automation (automated steering and accelerating/braking simultaneously).

\section{METHOD}

\subsection{Participants}

Participants $(N=300)$ were recruited from the online research service Prolific.ac. Twenty-four additional potential participants began the study but declined to complete the survey or did not finish within the allotted time frame. Participants were required to be 18 years old or older, to be currently residing in the United States, and to have previously reported being a car owner. An additional 10 participants were dropped from further analyses for self-reporting inattentiveness on an attention check question. The final sample used in analysis was 290 participants (128 female, 161 male, 1 who preferred not to respond; $M$ age $=33.16$ years, $S D=10.53$, $m d n=31$, age data missing for 5 participants). Participants were paid $\$ 2.00$ to complete the online survey. Data collection took place from June 21-22, 2021 and used a Qualtrics survey. 


\subsection{Procedure}

After informed consent, participants were randomly assigned to read one of two descriptions of a driver monitoring system. In the manual driving condition, the description did not mention automation, which implied that manual driving will be monitored. In the Level 2 (L2) automation condition, the description had an additional description of a L2 advanced driver assistance system (ADAS) (see [17]). The description indicated that the ADAS can control steering and accelerating/braking in some circumstances, and that the driver is required to oversee the vehicle at all times. The L2 description indicated that DM would be used only when the ADAS was engaged.

Next, all participants responded to questions about nine potential driving distractions: listening to music, talking to a passenger, looking out the window at scenery for longer than two seconds, taking both hands off of the steering wheel, having a handsfree phone conversation, eating or drinking, reading or browsing information on a smartphone, typing or entering information on a smartphone, and driving while in an adverse mental state. These distractions represented a sample of the activities that drivers expect to be able to engage in during periods of automated driving (see, e.g., [15]). Potential distractions that could be considered necessary for the driving task (e.g., interacting with the vehicle console to input a GPS destination) were not examined here, in part due to practical constraints on the number of questions that could fit into a brief online survey.

Participants rated the perceived reasonableness and perceived risk they ascribed to each potential distraction (for a similar approach see [14]). Participants also rated the perceived fairness of four possible consequences when a DM system detects each distraction: doing nothing, providing an alert to the driver, locking out (preventing) the driver from accessing features or devices unrelated to driving, and reporting distracted driving to the driver's insurance company. All ratings were made on scales from 1 to 7 with anchors worded to reflect the target constructs (see Figure 1 for verbatim anchors for each rating scale). Questions about distractions stated that the distraction occurred "while driving" in the manual driving condition or "while driving with the advanced driver assistance system engaged" in the L2 condition. Since the Level 2 condition indicated automation was present, two additional consequences were rated only in the L2 condition: locking out (preventing) the driver from using the advanced driver assistance features and taking over control of the vehicle. For each distraction, questions about reasonableness/risk were presented together, as were questions about perceived fairness. Whether reasonableness/risk or fairness were rated first was randomly determined for each participant, and within these blocks, the order of presentation of distractions was randomly determined. At the end of the survey, participants answered a series of demographic and driving background questions and were debriefed.

\section{RESULTS}

This research was exploratory, so results are presented in a descriptive format. Figure 1 shows the distributions of ratings (combined across the manual driving and L2 automation conditions) of reasonableness, risk, and fairness of potential consequences of DM detecting each of the distractions. Figure 2 shows the mean ratings comparing the manual driving condition to the L2 automation condition. Figure 3 shows the distributions of responses and mean ratings for the two automation-specific consequences that only applied to the L2 automation condition. For figures comparing manual driving to $L 2$ automated driving, $95 \%$ confidence intervals are shown to help facilitate the inferences that are of interest to the reader (see [3]). Exploratory correlations 
showed that, collapsed across all distractions, perceived reasonableness and risk were both moderate to strong predictors of support for DM consequences ( $r s=.27$ to .57 ).

\section{DISCUSSION AND CONCLUSIONS}

Drivers showed considerable variability in their perceptions of the risk and reasonableness associated with various driving distractions, as well as in the perceived fairness of consequences when DM systems detect distraction (see Figures 1 and 3). This suggests that designing a generally acceptable DM system will be challenging, as drivers have different expectations regarding what constitutes risk and a fair approach to monitoring. Perceived fairness of driver monitoring consequences was linked to perceived risk of distractions, but some behaviors that are known to have negative effects on driving (e.g., handsfree phone conversations [18], and possibly even listening to music [2]) were perceived by most drivers to be relatively reasonable and low in risk. More research is needed to understand the best ways to calibrate DM feedback and consequences with regard to drivers' expectation about fairness, especially when drivers' perceived risk of the monitored behavior is inaccurate.

In general, across both manual driving and L2 automation scenarios, to the extent that the distraction was perceived to be risky and/or unreasonable, drivers tended to feel that alerts were a fair response when DM detects distraction (see Figure 1). More severe consequences such as locking out devices and features unrelated to driving were viewed less favorably overall, and drivers generally were averse to the possibility that a DM system would report distracted driving to their insurance company. Still, even these more severe consequences were viewed by some participants as a fair consequence for distractions perceived as being riskier-especially reading a smartphone screen, typing on a smartphone screen, or taking both hands off of the wheel.

Figure 1 also showed a trend suggesting that a DM system doing nothing (providing no alert or consequence) was perceived as less fair as the perceived risk of the distraction increased. Like with other applications of automation, managing trust and expectations following DM failures (including misses) likely will present challenges. Still, some drivers felt that DM doing nothing (providing no alert or consequence) was completely fair for all distractions, which suggested that some drivers may view any DM consequence as an imposition.

Comparing DM during manual driving versus $L 2$ automated driving, patterns of responses generally followed similar trends (see Figure 2). For risk perception, mean ratings of riskiness were higher for manual driving than for L2 automated driving for all distractions; visual inspection of confidence intervals suggested that some (but not all) of these differences were statistically meaningful (see, e.g., [3] for a tutorial on using confidence intervals for visual statistical inferences with independent group means). This suggested that risk tolerance for distractions is at least slightly greater during periods of automation, which aligns with anecdotal reports of automation abuse. It seems likely that, despite warnings that drivers must monitor Level 2 automation, drivers perceive distractions as less risky when automation is engaged, although this effect may be small and may depend upon the specific distraction. Visual inspection of the graphs in Figure 2 also suggested that drivers will view some distractions differently during manual driving versus L2 automated driving, but more research is needed to understand these differences. More severe automation-specific consequences (lockout of ADAS and involuntary takeover) were viewed generally unfavorably by most drivers (see Figure 3), though again, distractions such as smartphones that are perceived to be especially risky may represent an exception to this rule for at least some drivers. 
As an exploratory investigation, the results presented here should be viewed as suggestive and hypothesisgenerating rather than confirmatory. Since we appear to be on the cusp of ubiquitous deployment of driver monitoring systems, a crucial research need is to understand how to use the information from DM sensors and algorithms to collaborate with drivers toward the goal of increased safety. Very little appears to be known about driver perceptions and preferences regarding DM, and a better understanding of these issues likely will be critical for gaining trust and acceptance of DM systems from drivers. The current study suggested that the perceived fairness of DM may be affected by individual differences, the type of distraction detected and its perceived risk, the type of consequence, and even the presence or absence of automated functions in the vehicle. A good deal more research will be required to understand how to design DM systems to be perceived as mentoring and collaborative [6] rather than overbearing and punitive.
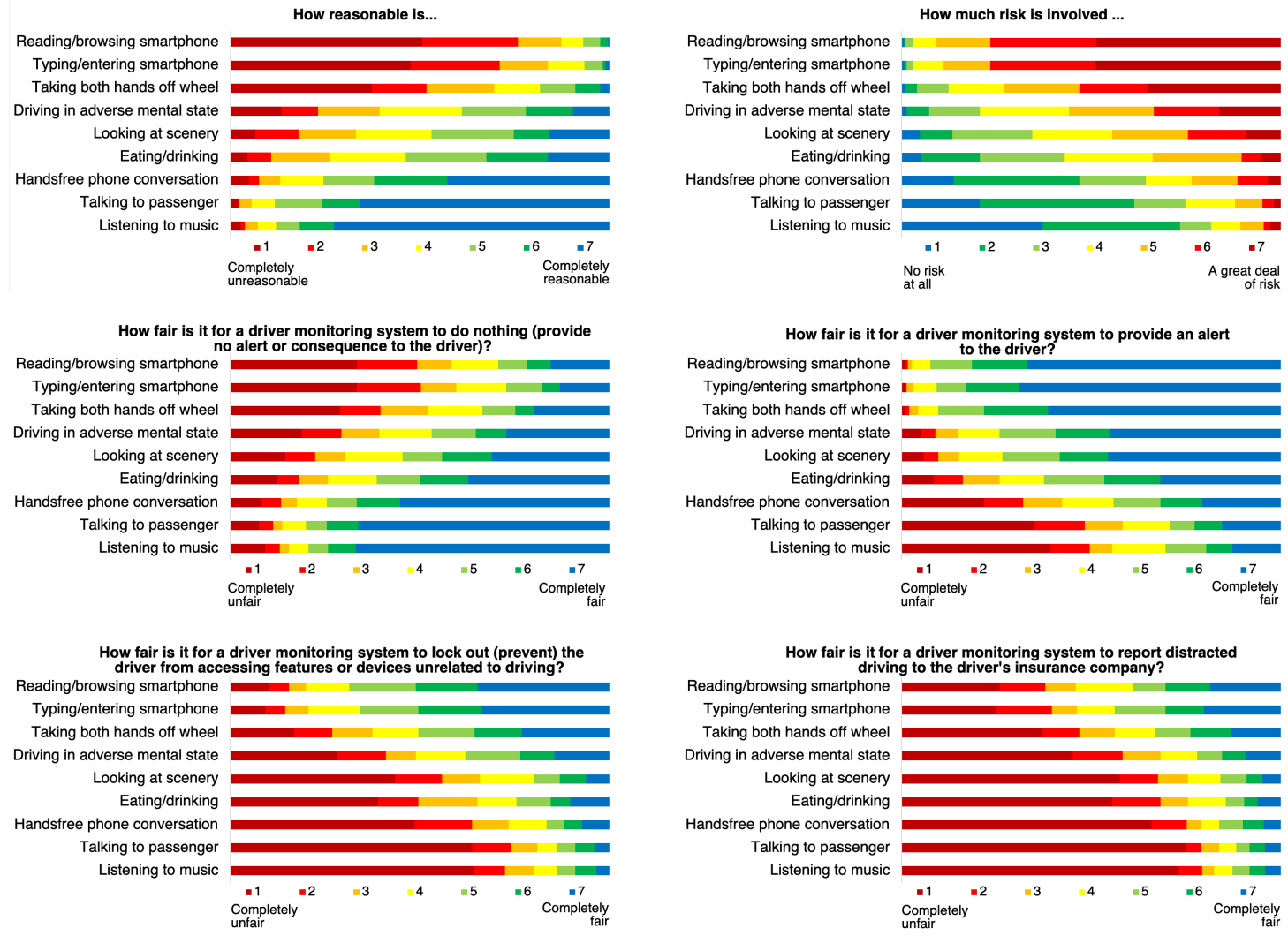

Figure 1. Distributions of ratings of perceived reasonableness, risk, and fairness of driver monitoring consequences for driving distractions. The top six panels are ratings for manual driving and Level 2 automation conditions combined $(N=$ 290). 
How reasonable is..

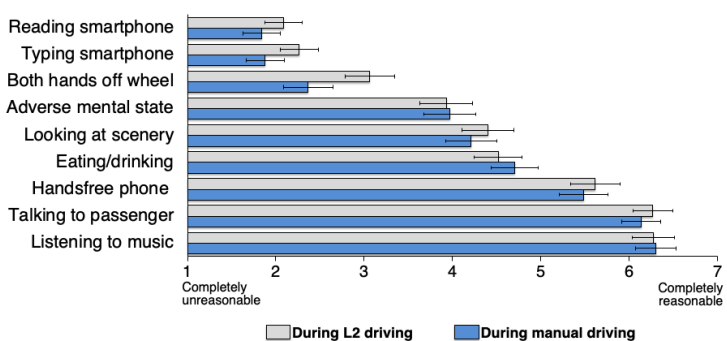

How fair is it for a driver monitoring system to do nothing (provide no

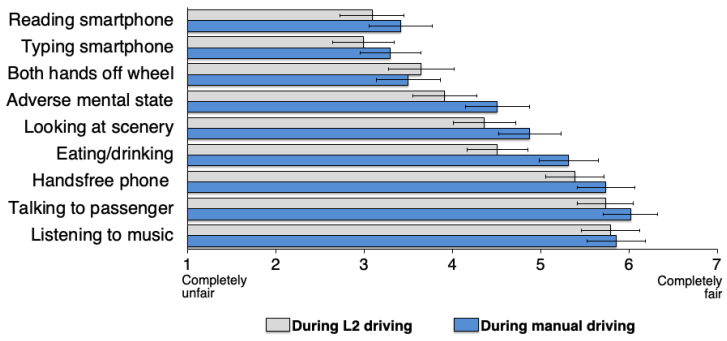

How fair is it for a driver monitoring system to lock out (prevent) the

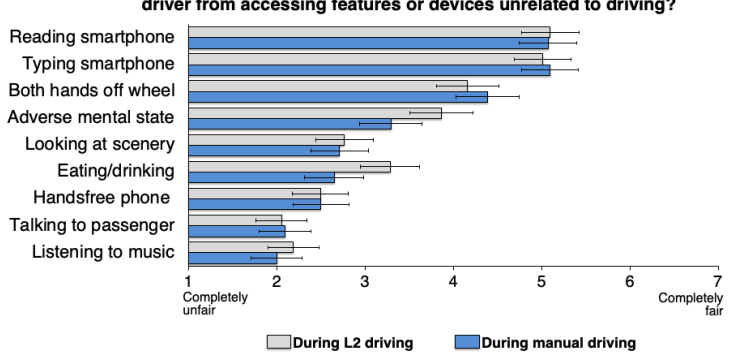

How much risk is involved...

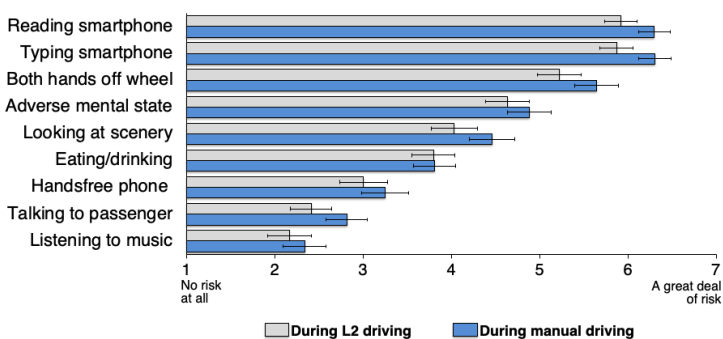
How fair is it for a driver monitoring system to provide an alert

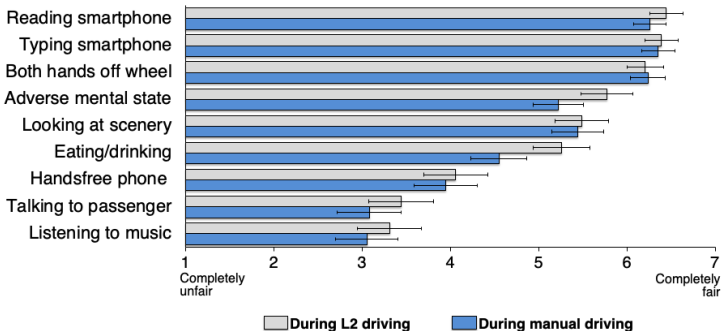

How fair is it for a driver monitoring system to report distracted

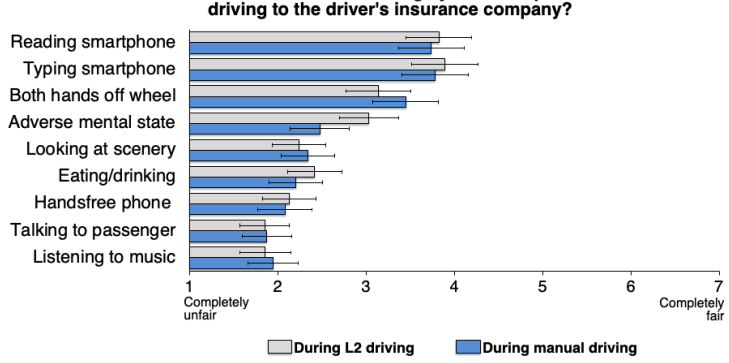

Figure 2. Mean ratings of perceived reasonableness, risk, and fairness of driver monitoring consequences for driving distractions comparing the manual driving $(n=147)$ and Level 2 automation ( $n=143$ conditions). Error bars represent $95 \%$ confidence intervals. 


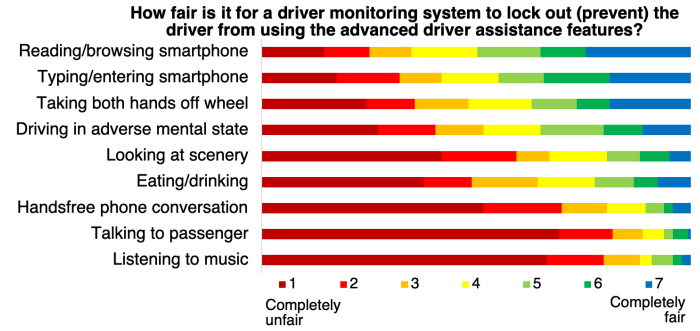

How fair is it for a driver monitoring system to lock out (prevent) the

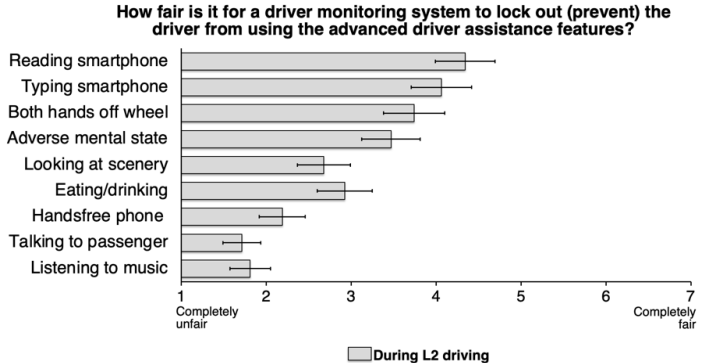

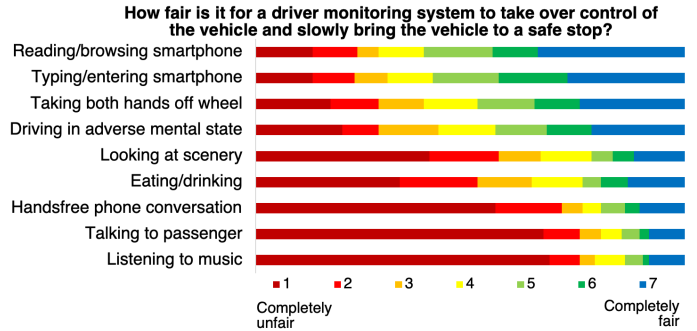

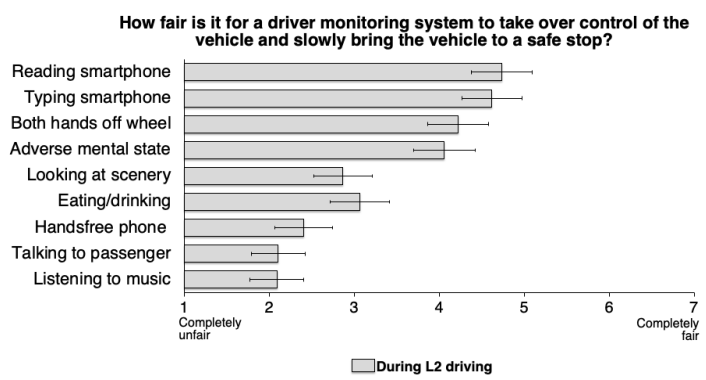

Figure 3. Distributions (upper panels) and mean ratings (lower panels) of perceived fairness of driver monitoring consequences for driving distractions for Level 2 automation-specific consequences $(n=143)$. Error bars represent $95 \%$ confidence intervals in the lower panels.

\section{ACKNOWLEDGMENTS}

This research was supported by funding from three internal sources at Lafayette College, including a Faculty Research Grant from the Academic Research Committee, funding from a Marquis Distinguished Teaching Award to the author, and funding from the Department of Psychology. Data, syntax, and supplemental materials are available at https://osf.io/npzru/.

\section{REFERENCES}

[1] G. Stoney Alder and Maureen L. Ambrose. 2005. An examination of the effect of computerized performance monitoring feedback on monitoring fairness, performance, and satisfaction. Organ. Behav. Hum. Decis. Process. 97, 2 (July 2005), 161-177. DOI:https://doi.org/10.1016/j.obhdp.2005.03.003

[2] Warren Brodsky and Zack Slor. 2013. Background music as a risk factor for distraction among youngnovice drivers. Accid. Anal. Prev. 59, (2013), 382-393.

[3] Geoff Cumming and Sue Finch. 2005. Inference by Eye: Confidence Intervals and How to Read Pictures of Data. Am. Psychol. 60, 2 (2005), 170-180.

[4] Birsen Donmez, Linda Ng Boyle, and John D. Lee. 2006. The Impact of Distraction Mitigation Strategies on Driving Performance. Hum. Factors J. Hum. Factors Ergon. Soc. 48, 4 (December 2006), 785-804. DOI:https://doi.org/10.1518/001872006779166415

[5] Birsen Donmez, Linda Ng Boyle, and John D. Lee. 2008. Mitigating driver distraction with retrospective and concurrent feedback. Accid. Anal. Prev. 40, 2 (March 2008), 776-786. DOI:https://doi.org/10.1016/j.aap.2007.09.023

[6] Mahtab Ghazizadeh and John D. Lee. 2018. Modelling driver acceptance: From feedback to monitoring and mentoring systems. In Driver Acceptance of New Technology. CRC Press, 51-70. 
[7] William J. Horrey, Mary F. Lesch, Marvin J. Dainoff, Michelle M. Robertson, and Y. Ian Noy. 2012. OnBoard Safety Monitoring Systems for Driving: Review, Knowledge Gaps, and Framework. J. Safety Res. 43, 1 (February 2012), 49-58. DOI:https://doi.org/10.1016/j.jsr.2011.11.004

[8] Alaa El Khatib and Fakhri Karray. 2020. Driver Inattention Detection in the Context of Next-Generation Autonomous Vehicles Design: A Survey. IEEE Trans. Intell. Transp. Syst. 21, 11 (2020), 14.

[9] Timothy B. Lee. 2021. Legislation would mandate driver-monitoring tech in every car. Ars Technica. Retrieved July 1, 2021 from https://arstechnica.com/tech-policy/2021/04/legislation-would-mandatedriver-monitoring-tech-in-every-car/

[10] Tim Levin. 2021. Tesla Autopilot Issues Are Part of Messy Transition for Auto Industry. Retrieved July 5, 2021 from https://www.businessinsider.com/tesla-autopilot-issues-assistance-software-automakersmarketing-driver-education-experts-2021-6

[11] Cade Metz. 2021. The Costly Pursuit of Self-Driving Cars Continues On. And On. And On. The New York Times. Retrieved July 5, 2021 from https://www.nytimes.com/2021/05/24/technology/self-drivingcars-wait.html

[12] Robert H Moorman and Deborah L Wells. Characteristics, Perceived Fairness, and Job. 15.

[13] Raja Parasuraman and Victor Riley. 1997. Humans and automation: Use, misuse, disuse, abuse. Hum. Factors 39, 2 (June 1997), 230-253. DOI:https://doi.org/10.1518/001872097778543886

[14] Jayesh Patel, David J. Ball, and Huw Jones. 2008. Factors influencing subjective ranking of driver distractions. Accid. Anal. Prev. 40, 1 (January 2008), 392-395.

DOI:https://doi.org/10.1016/j.aap.2007.07.006

[15] Bastian Pfleging, Maurice Rang, and Nora Broy. 2016. Investigating user needs for non-driving-related activities during automated driving. In Proceedings of the 15th International Conference on Mobile and Ubiquitous Multimedia - MUM '16, ACM Press, Rovaniemi, Finland, 91-99.

DOI:https://doi.org/10.1145/3012709.3012735

[16] Benjamin Preston. 2021. Call for Driver Monitoring Systems to Improve Safety - Consumer Reports. Consumer Reports. Retrieved May 20, 2021 from https://www.consumerreports.org/car-safety/call-fordriver-monitoring-to-improve-car-safety/

[17] SAE On-road Automated Vehicles Standards Committee. 2018. SAE J3016: Taxonomy and Definitions for Terms Related to On-Road Motor Vehicle Automated Driving Systems.

[18] David L. Strayer and William A. Johnston. 2001. Driven to Distraction: Dual-Task Studies of Simulated Driving and Conversing on a Cellular Telephone. Psychol. Sci. 12, 6 (November 2001), 462-466. DOI:https://doi.org/10.1111/1467-9280.00386

[19] Phillip Taylor, Nathan Griffiths, Abhir Bhalerao, Zhou Xu, Adam Gelencser, and Thomas Popham. 2015. Warwick-JLR driver monitoring dataset (DMD): statistics and early findings. In Proceedings of the 7th International Conference on Automotive User Interfaces and Interactive Vehicular Applications (AutomotiveUI '15), Association for Computing Machinery, New York, NY, USA, 89-92. DOI:https://doi.org/10.1145/2799250.2799286

[20] Patrick Tchankue, Janet Wesson, and Dieter Vogts. 2011. The impact of an adaptive user interface on reducing driver distraction. In Proceedings of the 3rd International Conference on Automotive User Interfaces and Interactive Vehicular Applications - AutomotiveUI '11, ACM Press, Salzburg, Austria, 87. DOI:https://doi.org/10.1145/2381416.2381430

[21] Joel S. Warm, Raja Parasuraman, and Gerald Matthews. 2008. Vigilance requires hard mental work and is stressful. Hum. Factors 50, 3 (June 2008), 433-441. DOI:https://doi.org/10.1518/001872008X312152

[22] Ji Hyun Yang, Hyeon-Bin Jeong, Jihyuck Han, and Sejoon Lim. 2017. Driver State Estimation Based on Dynamic Bayesian Networks Considering Different Age and Gender Groups. In Proceedings of the 9th International Conference on Automotive User Interfaces and Interactive Vehicular Applications Adjunct (AutomotiveUI '17), Association for Computing Machinery, New York, NY, USA, 131-135. DOI:https://doi.org/10.1145/3131726.3131752

[23] Road safety: Commission welcomes agreement on new EU rules to help save lives. European Commission - European Commission. Retrieved July 2, 2021 from https://ec.europa.eu/commission/presscorner/detail/en/IP_19_1793 